\title{
MAPS AND CHARTs
}

MAPS

1. Distribution of uniserial harpoons and wavy line pottery

2. Diffusion of Nostratic

3. Diffusion of Afroasiatic

3a. From Asia, Militarev and Schnirelman

3b. From Africa, Diakonoff

3c. - Orel and Stolbova.

3d. $\longrightarrow$, Ehret.

3e. $—$ Blench

3f. $\longrightarrow$, Bender

3g. - Bernal, 1980

3h. —, Bernal, 2004

4. Diffusion of Indo-European

5. Ancient East Mediterranean

6. Southern Greece

7. Boiotia.

\section{Charts}

1. Indo-Hittite Language Family

2. Egyptian Chronology

3. Aegean Chronology

4. Greek Chronology 


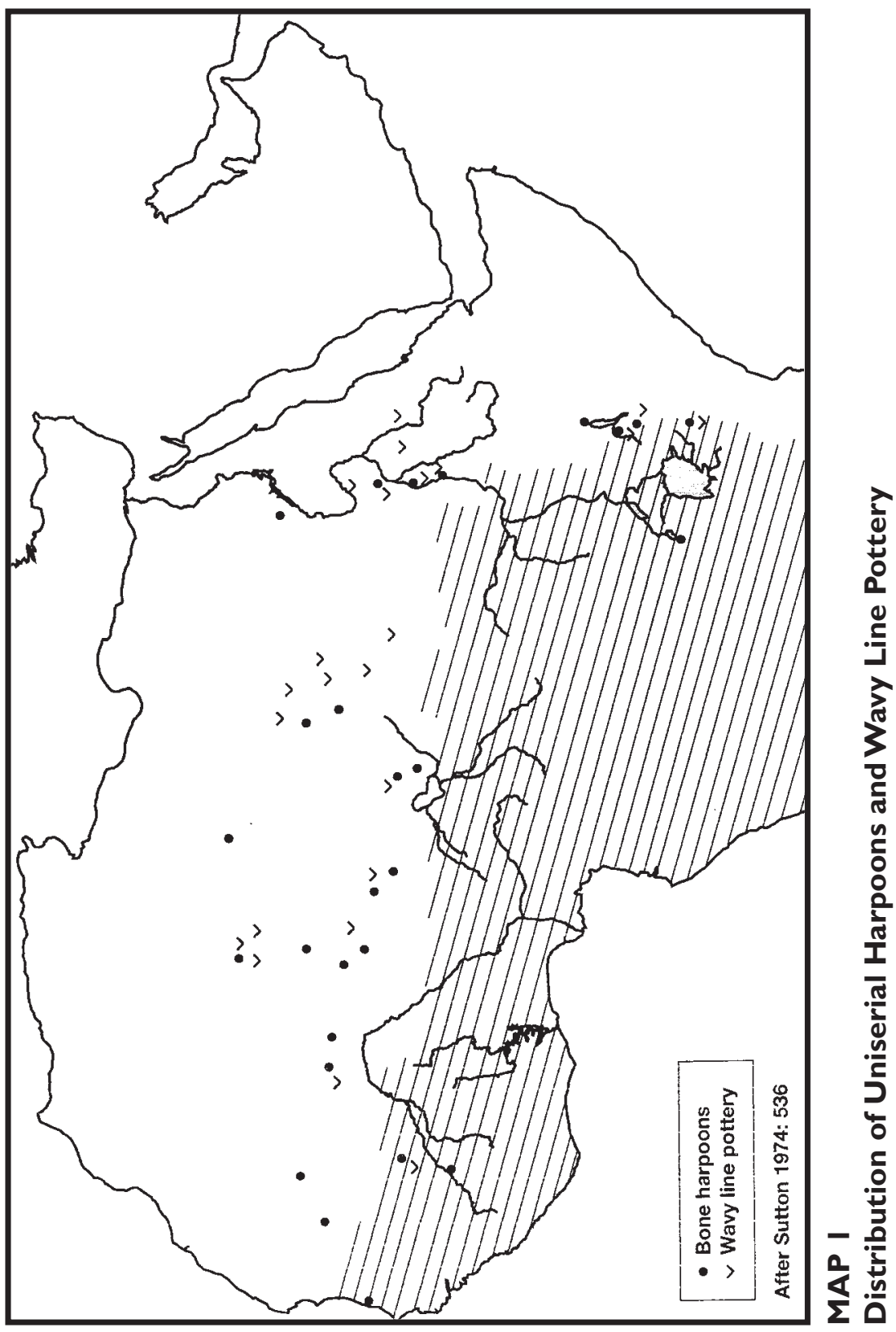




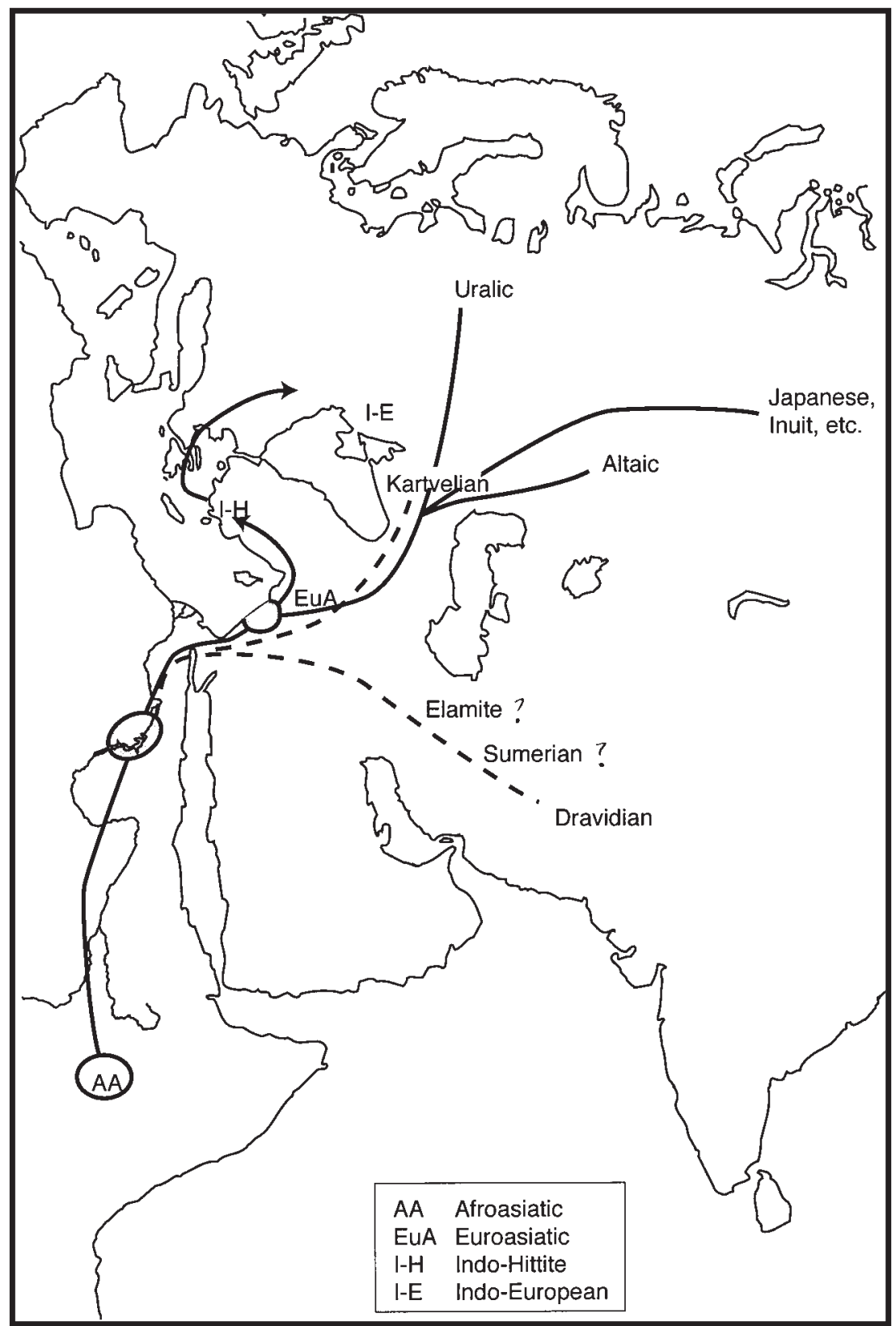

MAP 2

Diffusion of Nostratic 


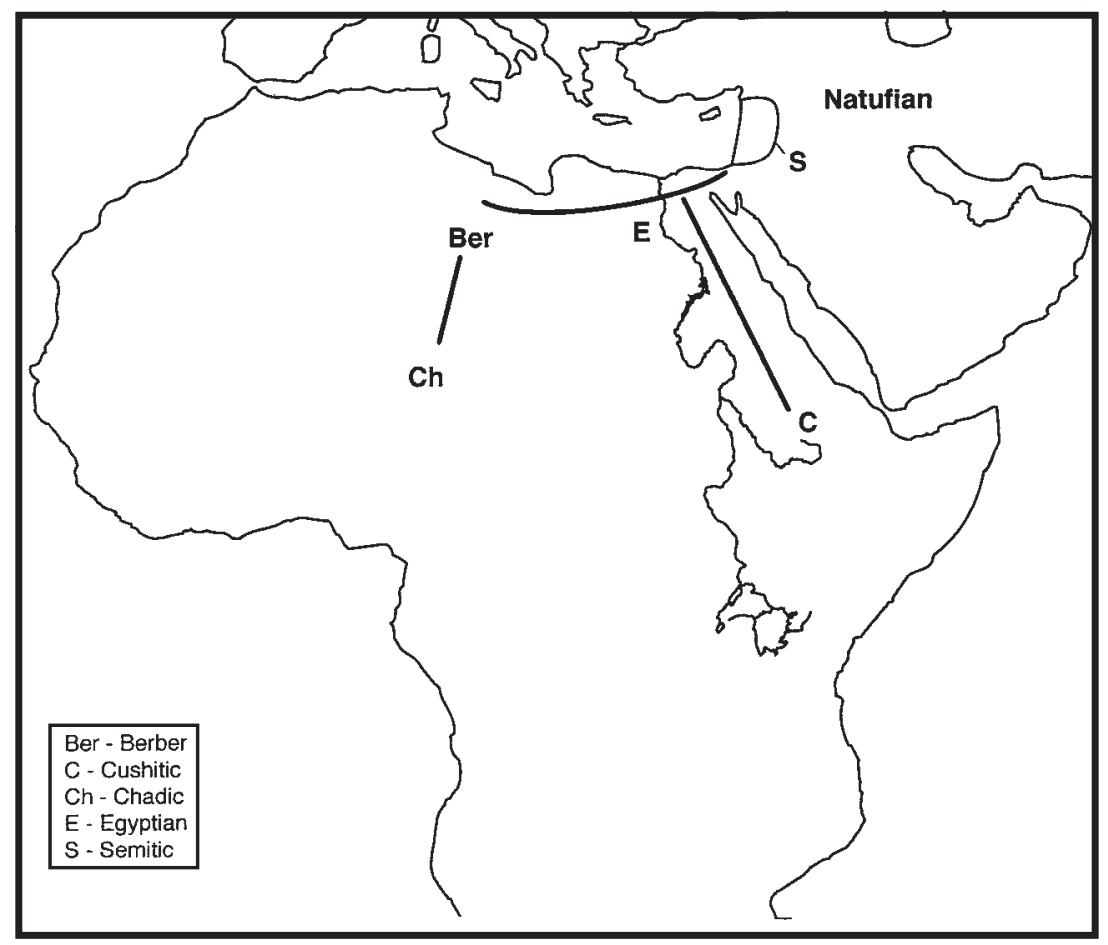

MAP 3a

Diffusion of Afroasiatic: From Asia, Militarev and Schnirelman 


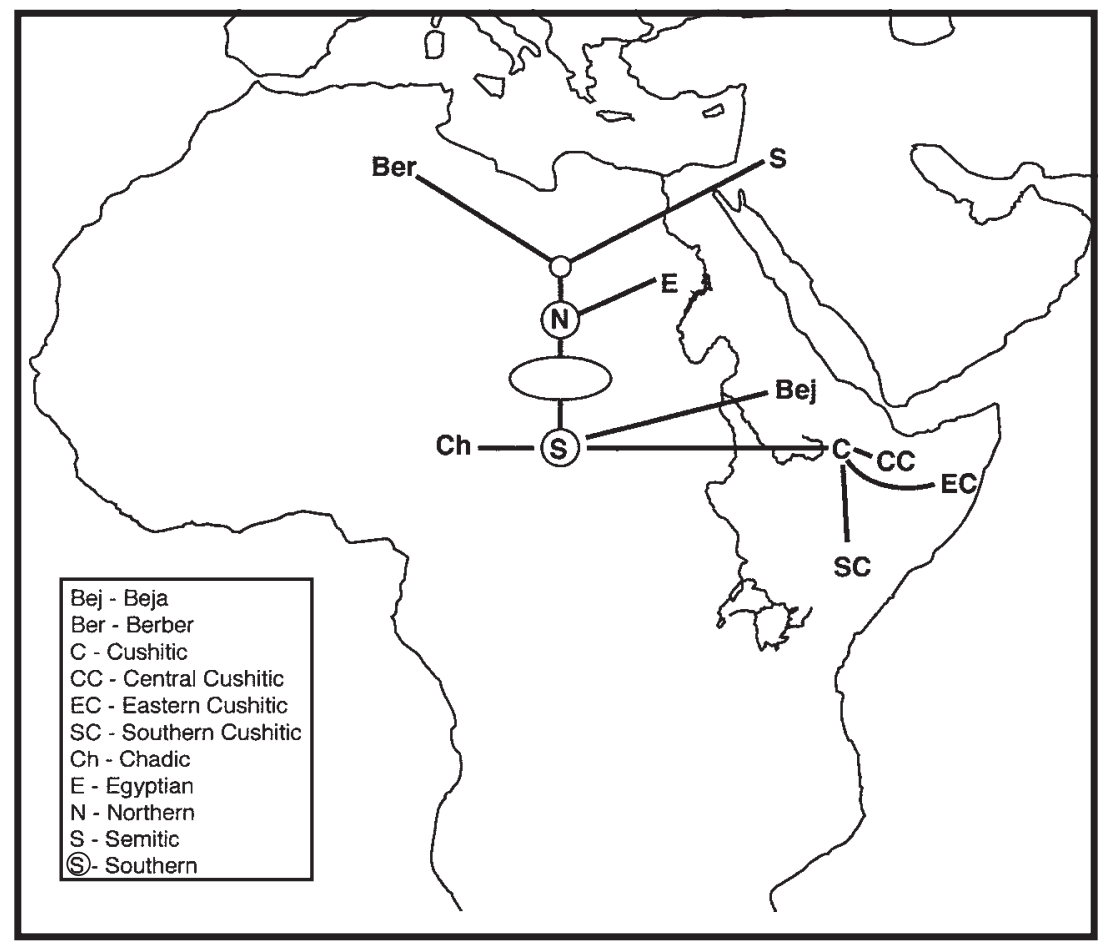

\section{MAP 3b}

Diffusion of Afroasiatic: From Africa, Diakonoff 


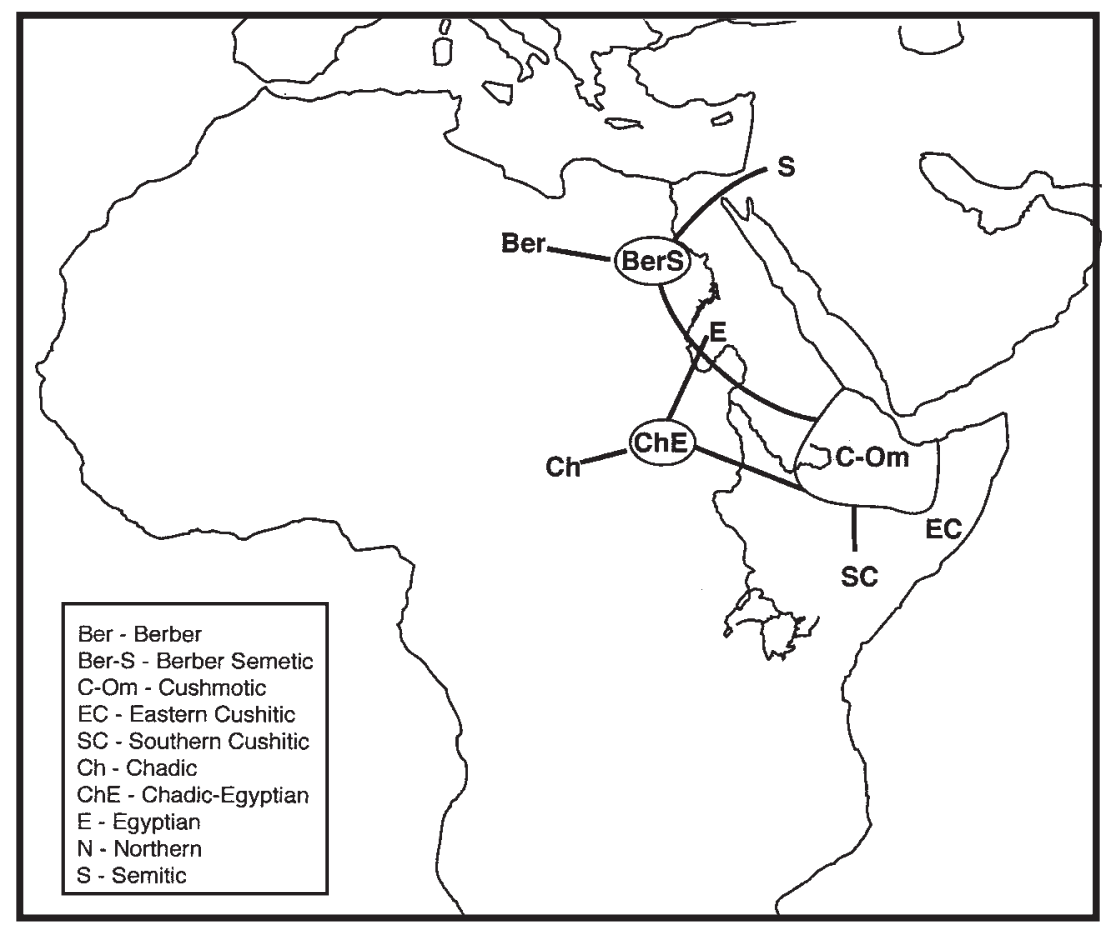

MAP 3c

Diffusion of Afroasiatic: From Africa, Orel and Stolbova 


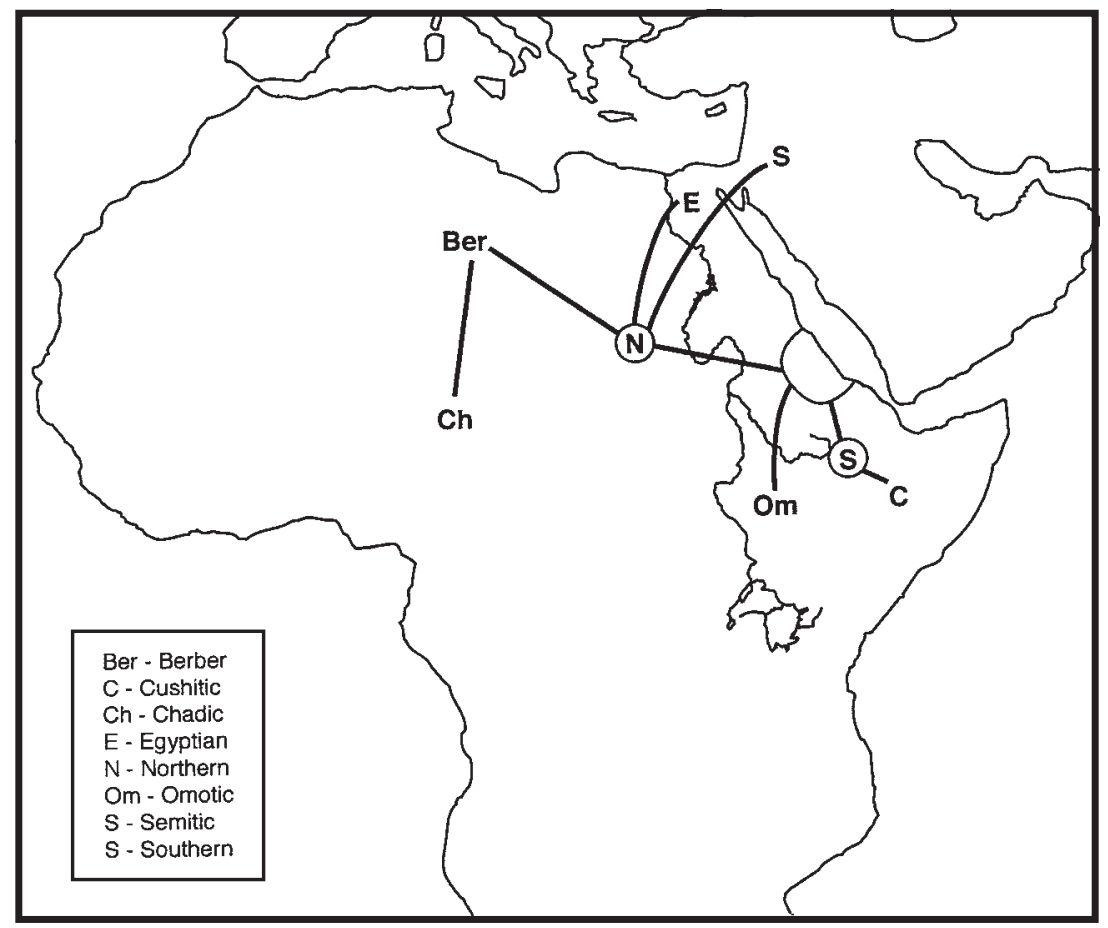

MAP 3d

Diffusion of Afroasiatic: From Africa, Ehret 


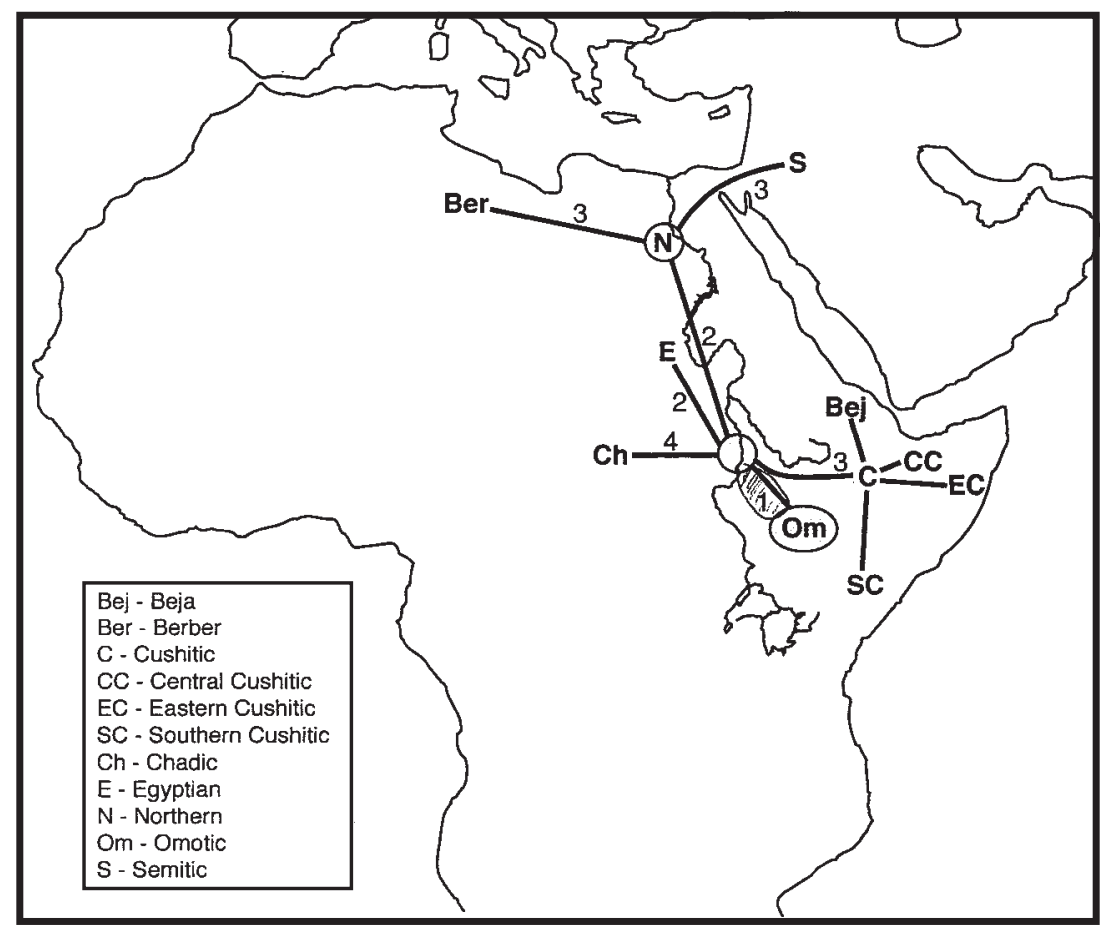

MAP $3 e$

Diffusion of Afroasiatic: From Africa, Blench 


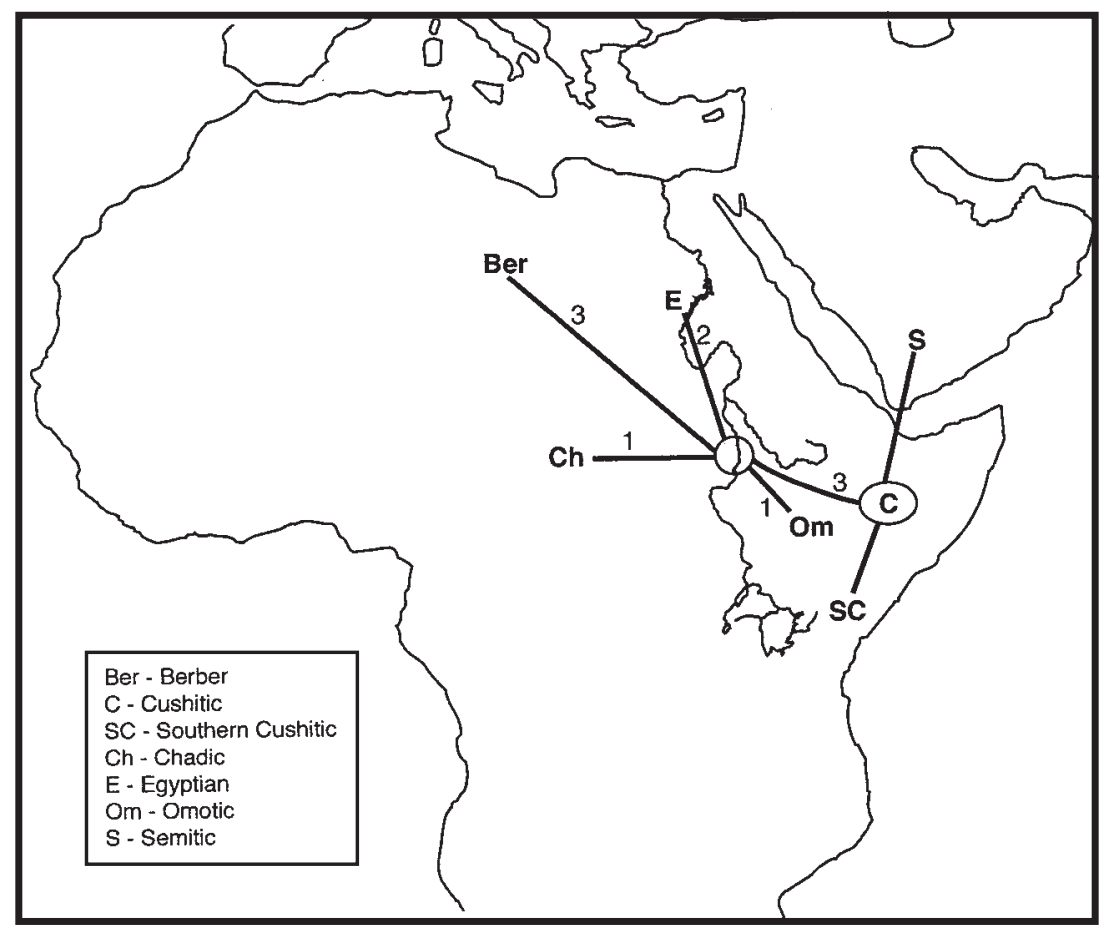

\section{MAP $3 f$}

Diffusion of Afroasiatic: From Africa, Bender 


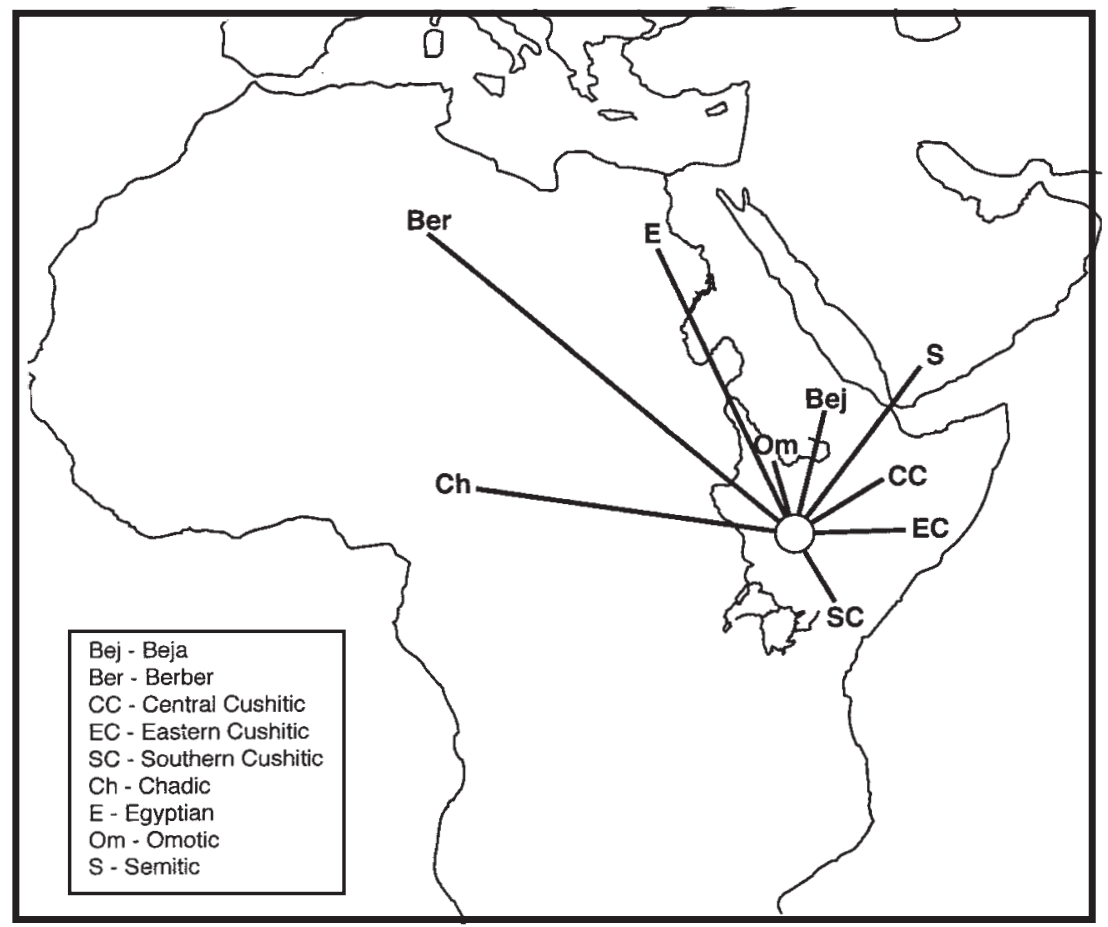

MAP 3g

Diffusion of Afroasiatic: From Africa, Bernal, 1980 


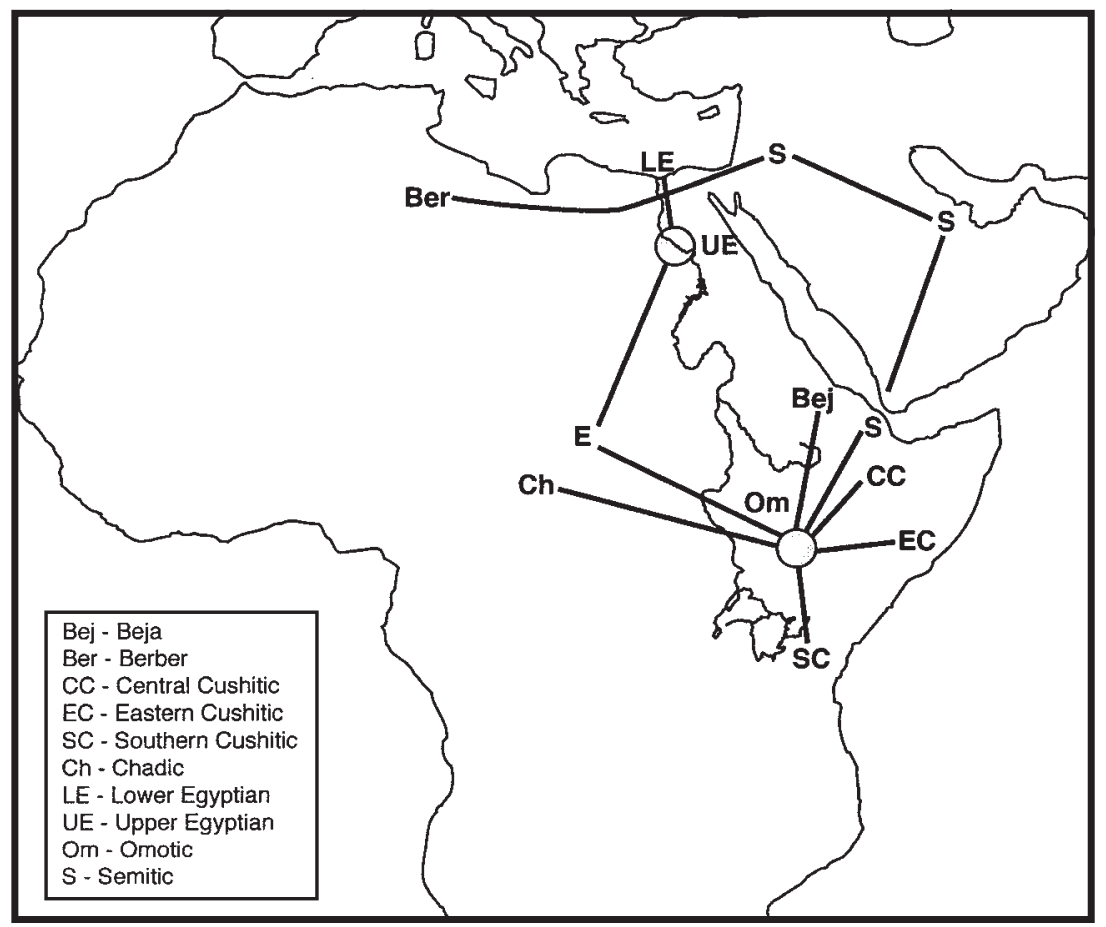

MAP $3 h$

Diffusion of Afroasiatic: From Africa, Bernal, 2004 


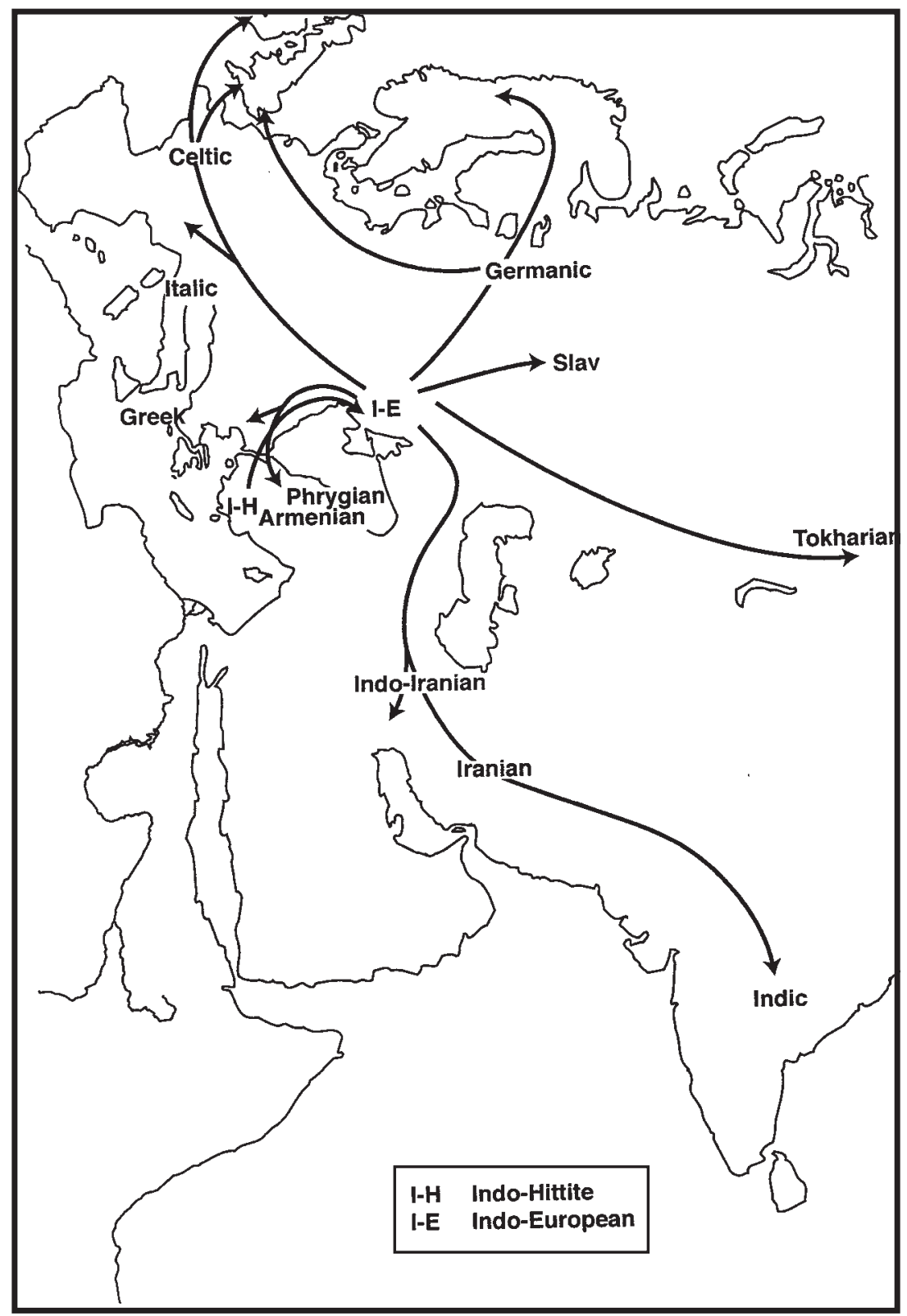

MAP 4

The Diffusion of Indo-European 


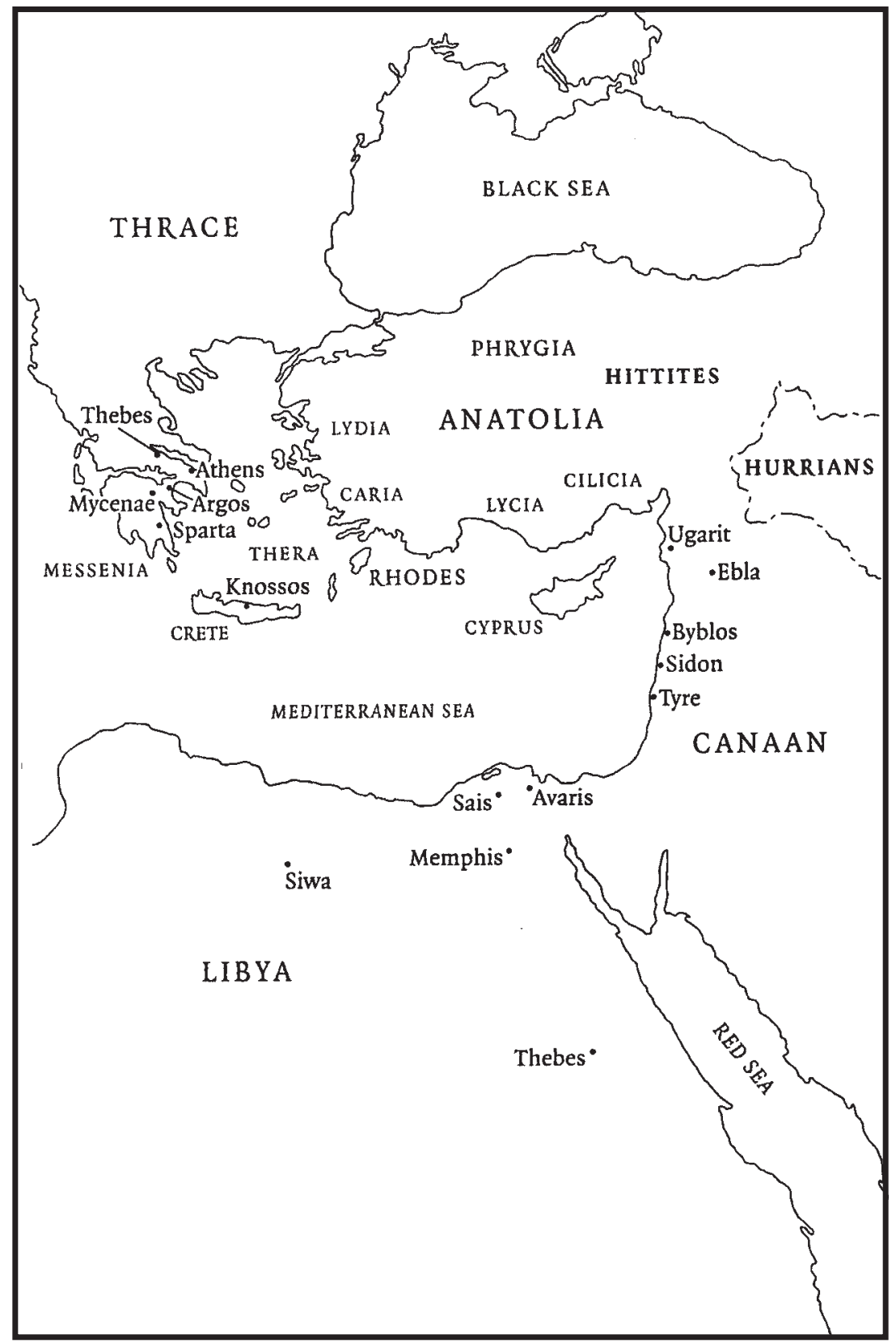

MAP 5

Ancient East Mediterranean 


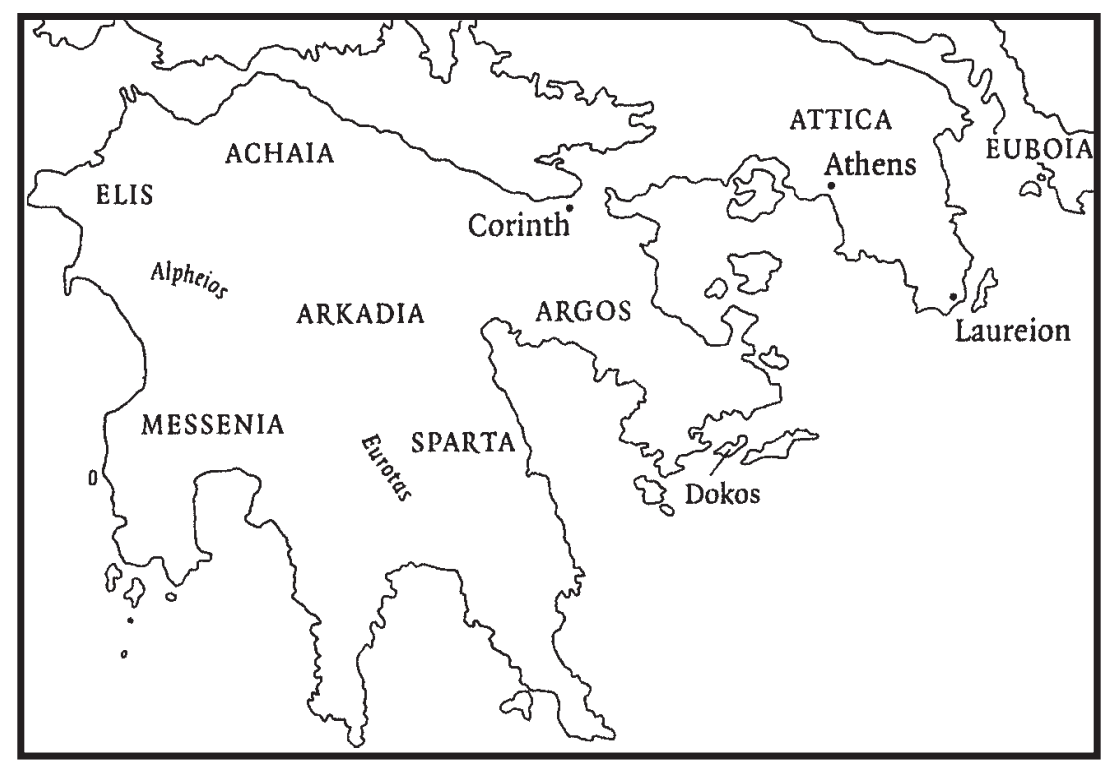

MAP 6

\section{Southern Greece}

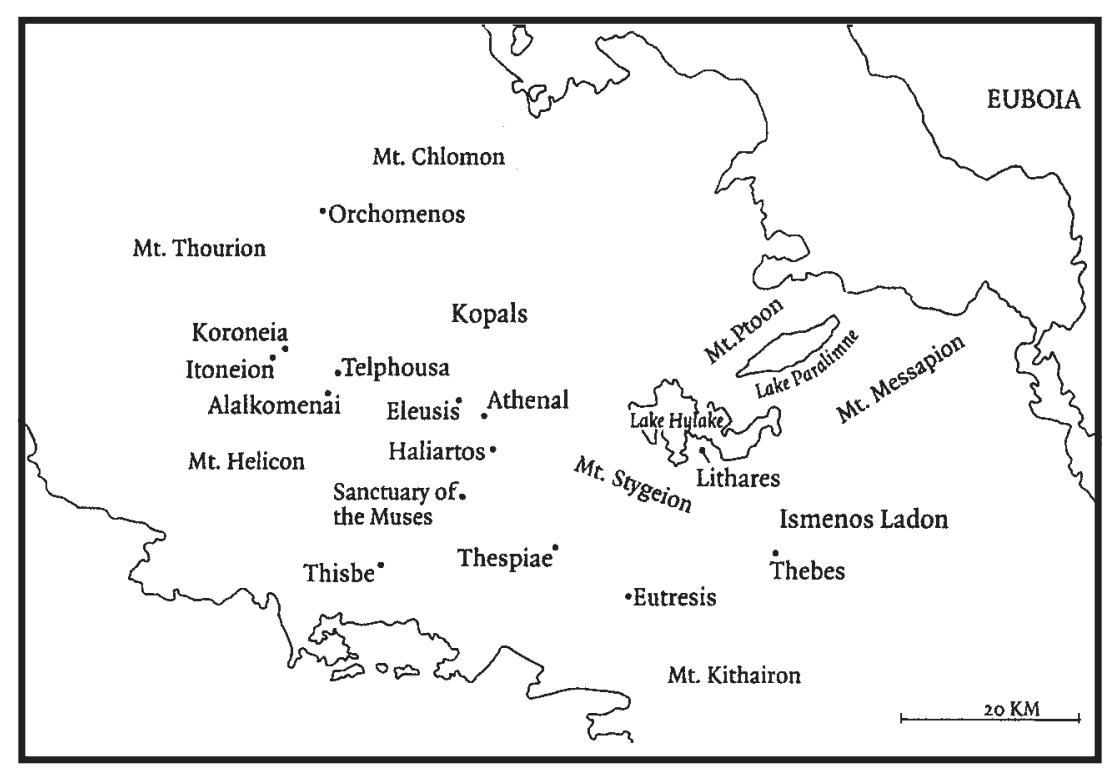

MAP 7

\section{Boiotia}




\section{CHART I}

\section{Indo-Hittite Language Family}

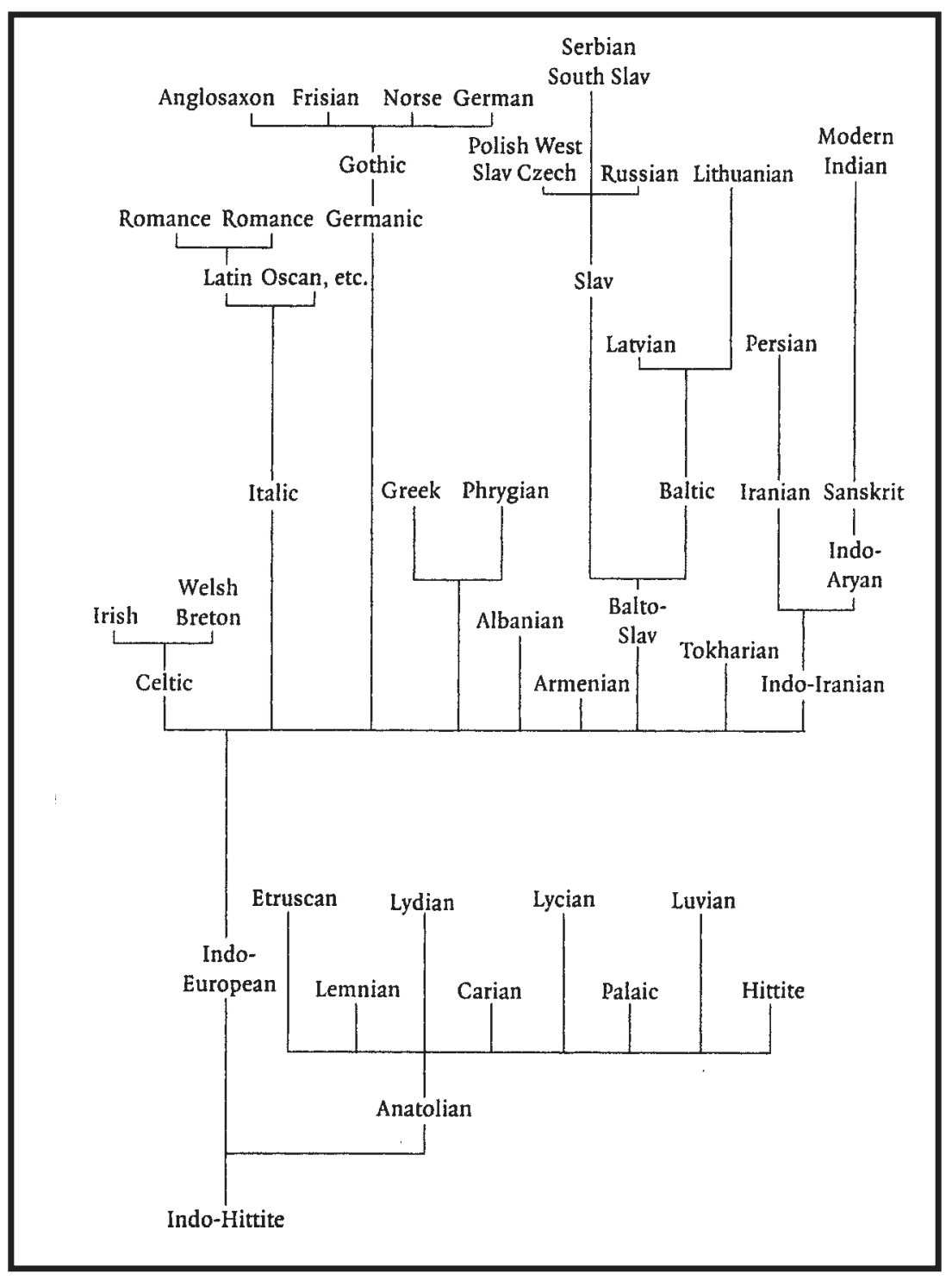


xxxvi

BLACK ATHENA

CHART 2

Egyptian Chronology

\begin{tabular}{ccccccc}
\hline Dynasty & Breasted & Meyer & CAH & Helck & Mellart & Bernal \\
\hline 1st & 3400 & $3315 \pm 100$ & 3100 & 2955 & 3400 & 3400 \\
2nd & & & 2900 & 2780 & 3200 & 3200 \\
3rd & 2980 & $2895 \pm 100$ & 2730 & 2635 & 2950 & 3000 \\
4th & 2900 & $2840 \pm 100$ & 2613 & 2570 & 2850 & 2920 \\
5th & 2750 & $2680 \pm 100$ & 2494 & 2450 & 2725 & 2800 \\
6th & 2625 & $2540 \pm 100$ & 2345 & 2290 & 2570 & 2630 \\
7th & 2475 & - & 2181 & 2155 & 2388 & 2470 \\
8th & 2475 & - & - & - & 2388 & 2470 \\
9th & 2445 & $2360 \pm 100$ & 2160 & - & - & 2440 \\
10th & - & - & 2130 & - & - & - \\
11 th & 2160 & 2160 & 2133 & 2134 & 2287 & 2140 \\
12th & 2000 & $2000 / 1997$ & 1991 & 1991 & 2155 & 1979 \\
13th & 1788 & 1778 & 1786 & $?$ & 1946 & 1801 \\
14th & - & - & - & - & - & - \\
15th & - & - & 1674 & 1655 & 1791 & 1750 \\
16th & - & - & 1684 & - & - & - \\
17th & - & - & - & - & - & - \\
18th & 1580 & $1580 / 75$ & 1567 & 1552 & 1567 & 1567 \\
19th & 1315 & 1320 & 1320 & 1306 & 1320 & 1320 \\
20th & 1200 & 1200 & 1200 & $1196 / 86$ & 1200 & 1200 \\
\hline
\end{tabular}

Sources: Breasted (19o6, I, pp. 40-5); Meyer (197ob, pp. 68 and 178); Cambridge Ancient History (charts at the end of vols I.2B, II. 1 and II.2); Helck (1971, chart; 1979, pp. 146-8); Mellaart (1979, pp. 9 and 19). 
CHART 3

Aegean Chronology

\begin{tabular}{|c|c|c|c|c|c|}
\hline $\begin{array}{l}\text { Ceramic } \\
\text { Period }\end{array}$ & CAH & $K \mathcal{E} M$ & Bet. & Bernal 1 & Bernal 2 \\
\hline EMI & $3000 ?$ & & & & 3300 \\
\hline EMII & $2500 ?$ & & & & 3000 \\
\hline EMIII & 2200 & & & & 2400 \\
\hline MMIA & 1900 & & & & 2050 \\
\hline MMIB & & 2000 & & & 1950 \\
\hline MMII & 1800 & & & & 1820 \\
\hline MMIII & 1700 & $1775-50$ & & 1730 & 1730 \\
\hline LMIA & 1600 & $1675-50$ & & 1650 & 1675 \\
\hline LHI & 1550 & & & & \\
\hline LMIB/LHIIA & 1500 & $1600-1575$ & 1610 & 1550 & 1600 \\
\hline LMII & 1450 & $1500-1475$ & 1550 & 1450 & 1520 \\
\hline LHIIB & 1430 & 1550 & & & 1520 \\
\hline LHIIIA1 & 1400 & & 1490 & & 1470 \\
\hline LMIIIA & 1380 & & 1490 & & 1470 \\
\hline \multicolumn{6}{|l|}{ LMIIIA2/ } \\
\hline LHIIIA2 & & & $1430-10$ & & 1410 \\
\hline \multicolumn{6}{|l|}{ LMIIIB / } \\
\hline LHIIIB & 1275 & $1375-50$ & 1365 & & 1370 \\
\hline \multicolumn{6}{|l|}{ LMIIIC/ } \\
\hline LHIIIG & 1180 & & 1200 & & 1220 \\
\hline
\end{tabular}

$\mathrm{CAH}=$ Cambridge Ancient History, 3 rd edition.

$\mathrm{K} \& \mathrm{M}=$ Kemp and Merrillees (1980) Minoan Potterv in Second Millennium Egypt.

Bet. $=$ Betancourt (1989) 'High chronology and low chronology: Thera archaeological evidence.'

Bernal 1 = Black Athena, Volume 1.

Bernal 2 = Black Athena, Volume 2 . 
xxxviii

BLACK ATHENA

\section{CHART 4}

\section{Greek Chronology}

Destruction of Thebes 1230-1225 BCE

Trojan War $1215^{-1205}$

Dorian and other invasions $115^{\circ-1} 120$

"Dark Ages"

Hesiod and Homer $105^{\circ}-85^{\circ}$

Geometric Ceramic period 9oo-750

Orientalizing period $75^{\circ}-65^{\circ}$

Archaic period $776-500$

Classical period $5^{\mathrm{OO}-320}$

Alexander and Hellenistic period 320-100

Roman 100 BCE-3OO CE 
Black Athena 
